\title{
Comentario: Problemas y mercadeo de las estatinas
}

\author{
THOMAS F. WHAYNE, Jr, MD, PhD
}

\section{Problems with statins and the marketing of these medications}

All statins inhibit hydroxymethylglutaryl Coenzyme A Reductase but each has a different chemical structure that may have individual advantages. Some pharmaceutical companies have minimized side effects and stated that dose has no relation to incidence. To the contrary, dose is related to side effects with all statins. Myopathy occurs in up to $10.5 \%$ of patients taking a high dose. There is an attempt to sell statins that have lost patent protection over-the-counter. However, evidence supports medical supervision as offering greatest patient safety. Concerns were raised about ezetimibe after the initial ENHANCE (efficacy) and SEAS (cancer risk) study but these concerns appear to have been answered. Fenofibrate can be used with a statin but gemfibrozil is contraindicated. Coenzyme Q-10 possibly helps to mitigate the risk of myopathy with a statin but evidence is not universally accepted. JUPITER represented a valid outcomes study but made a claim that rosuvastatin has special value in risk management because of decreased high sensitivity C-Reactive Protein. This actually occurs with any statin, a decrease also enhanced by ezetimibe. Statins have benefited the lives of our patients but, as with any treatment, the physician needs to look critically at all the problems and claims made.

(Rev Med Chile 2010; 138: 511-514).

Key words: Hydroxymethylglutaryl-Co A Reductase inhibitors; Hypercholesterolemia; Muscular diseases.

\section{Las guerras de las estatinas}

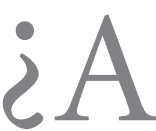

lguna estatina tiene alguna ventaja sobre otra? Parece que existen diferencias significativas de riesgo, siendo la cerivastatina una de ellas. En un estudio realizado en 2002, se registraron casos de muertes por rabdomiolisis en proporción a un millón de recetas $(3,16)$ para cerivastatina, siendo, posteriormente, retirada del mercado ${ }^{1}$. También se incluyen, en orden descendiente de mortalidad por un millón de recetas a la rabdomiolisis, lovastatina $(0,19)$, simvastatina $(0,12)$, atorvastatina $(0,04)$, pravastatina $(0,04)$ y fluvastatina $(0,00)$. La rosuvastatina no estaba disponible durante esa época. La lovastatina fue la primera estatina disponible $y$, luego de la aparición en 1991 de simvastatina ${ }^{2}$, las guerras de las estatinas empezaron, aun entre los diversos productos patentados por la misma empresa (lovastatina y simvastatina de Merck: observación personal). Las estatinas genéricas, ahora conocidas, son lovastatina, simvastatina y pravastatina; atorvastatina, fluvastatina y rosuvastatina todavía conservan su marca registrada. Todas las estatinas tienen una estructura química diferente a pesar de la inhibición de la hidroxilmetilglutaril-coenzima A reductasa ${ }^{3}$. Algunos pacientes desarrollarán efectos secundarios con alguna estatina y no con otra(s). Por consiguiente, para el médico, la disponibilidad a todas las estatinas es un boom, ya que ofrece la posibilidad de indicarle al paciente la que mejor tolere. Actualmente, la 
estatina genérica que parece ser más segura es la pravastatina. Esta llama la atención, en vista de los resultados de los estudios que se han hecho ${ }^{4}$ y por sus bajos precios: 4 dólares (copago) por mes en Walmart S.A. en los Estados Unidos de Norteamérica (EE.UU.) ${ }^{5}$.

\section{Problemas individuales con las estatinas}

A pesar de que la medicina le ha dado el nombre de "fármacos maravillosos" a las estatinas, y las compañías han declarado que éstas son perfectamente seguras, existen inconvenientes con su uso. Estos no reciben la cobertura necesaria en la literatura promocional ni en algunos estudios clínicos. En realidad, existen unos médicos y compañías farmacéuticas interesados en promocionar la venta sin receta de las estatinas. Actualmente, esta idea no parece ser muy buena por los riesgos inherentes, siendo uno de estos, el de aquellos pacientes carentes de suficiente educación o conciencia. De por sí, ya está documentado la cantidad de pacientes que no siguen adecuadamente las instrucciones escritas en las recetas ${ }^{6}$. Afortunadamente, la venta de las estatinas sin receta fue negada, por segunda vez, por la la Administración de Alimentos y Fármacos de los EE.UU., (FDA, siglas en inglés). Este dictamen se dio a pesar de que las metas estipuladas sobre las estatinas son buenas y los riesgos declarados no son graves. La adquisición de las estatinas sin una receta por parte de los pacientes de alto riesgo, sin seguir las órdenes médicas o cuidado médico regular, podría causar daños significativos, especialmente la rabdomiolisis, pudiendo pasar desapercibida y recibir tratamiento apropiado demasiado tarde $\mathrm{e}^{7,8}$.

Una compañía farmacéutica aun ha afirmado que su estatina a la dosis más alta no tiene más complicaciones que la recomendada a dosis más baja. No obstante, esta afirmación no es sustancial según los datos entregados a la FDA para la aprobación de cualquier estatina, ni tampoco una consideración de muchos estudios clínicos a más corto tiempo apoya tal afirmación ${ }^{9}$. Estudios prospectivos con información de observaciones específicas, muestran que más de $10 \%$ de los pacientes que toman una estatina en una dosis alta tienen síntomas de miopatía. En un estudio grande de 32.225 pacientes, $5,8 \%$ de los pacientes diabéticos y $6,7 \%$ de los pacientes no diabéticos desarrollaron mialgias relacionadas a las estatinas $^{10}$. En otro importante estudio observacional, Prediction of Muscular Risk in Observational Conditions (PRIMO), el cual incluyo 7.924 pacientes en Francia, que tomaban una estatina en altas dosis, 10,5\% de ellos desarrollaron síntomas musculares a partir de un mes del inicio de la estatina ${ }^{11}$.Así, con la información actualizada, se sugiere que la farmacocinética y las interacciones de las estatinas juegan un papel causal en la miopatía y que la miopatía tiene más relación a la dosis de la estatina que a la disminución de las LDL ${ }^{12}$.

Las estatinas aumentan las HDL, pero después de solamente seis semanas es posible observar una disminución del aumento inicial. En el caso de la atorvastatina, el aumento de las HDL logrado al principio puede disminuir a menos del nivel inicial con una dosis alta ${ }^{13}$.

\section{Problemas del uso de las estatinas con otros fármacos}

El uso adicional de la ezetimiba permite que una estatina permanezca a dosis baja y aparentemente más segura mientras se alcance una disminución marcada de las LDL (indicadores estándar de disminución de enfermedad coronaria (EC)). El beneficio de las estatinas para el paciente con alto riesgo cardiovascular (CV) sobrepasa cualquier riesgo. Sin embargo, el uso de tales fármacos debe ser cauteloso, especialmente en pacientes de mayor riesgo. Aunque actualmente hay mucho interés en la proteína $\mathrm{C}$ reactiva de alta sensibilidad (PCRas), solamente las LDL tienen evidencia médica sólida y confiable que apoya el hecho de que su disminución determina una reducción en la incidencia de episodios CVs agudos y crónicos. A pesar de la presión de utilizar estatinas a una dosis alta por parte de muchos médicos, la disminución de las LDL, al utilizar solo una estatina, se puede ver ya con la dosis inicial. Luego, cada vez que se dobla la dosis, en promedio, solamente ocurre una reducción de las LDL de $6 \%{ }^{14}$. Siendo las LDL el indicador estándar, la ezetimiba merece una consideración adicional, ya que es un fármaco capaz de disminuir las $\mathrm{LDL}^{15}$ hasta en $25 \%$ más, mantiendo de modo seguro bajas dosis y a largo palzo. El tratamiento de estatinas a altas dosis, como la atorvastatina, parece ser aceptable en algunos casos como en el síndrome coronario 
agudo $^{16}$. No obstante, la terapia a altas dosis de estatinas no generaran beneficios a largo plazo si se compara con el tratamiento a dosis más baja, ya que se alcanza el nivel de las LDL menor de $70 \mathrm{mg} /$ $\mathrm{dl}$ (valor deseable para el paciente con alto riesgo $\left.\mathrm{CV}^{17}\right)$. El estudio "Effect of Ezetimibe Plus Simvastatin Versus Simvastatin Alone on Atherosclerosis in the Carotid Artery" (ENHANCE) causó muchas preocupaciones acerca del uso de ezetimiba, pero evaluaciones cuidadosas no han demostrado ningún problema significativo en relación con este fármaco ${ }^{18}$. También, con el estudio, Simvastatin and Ezetimibe in Aortic Stenosis (SEAS) había una preocupación inicial acerca de un efecto de la ezetimiba en la incidencia del cáncer; un análisis posterior de este estudio y otros dos en progreso no demostraron una evidencia de efecto adversos propios de la ezetimiba ${ }^{19}$.

Otro asunto de interés médico en cuanto al uso de las estatinas, es el marcado aumento de miopatía y rabdomiolisis al combinar las estatinas con gemfibrozil ${ }^{20}$. Esta combinación debe evitarse y nunca debe ser usada. Muchas de las hiperlipidemias mixtas pueden manejarse con una estatina más ácido nicotínico, ácidos grasos omega-3 o fenofibrato ${ }^{21}$. Parece que los ácidos grasos omega-3 combinados con una estatina son especialmente seguros. Cuando se combina el fenofibrato e inclusive el ácido nicotínico con una estatina, es más seguro y de mayor ventaja mantener la dosis de la estatina lo más baja posible.

La coenzima Q-10 (CoQ) posiblemente ayuda a evitar la miopatía por parte de las estatinas o, por lo menos, disminuir los síntomas y dejar que el paciente continúe con estos fármacos $\mathrm{CVs}$ vitales $^{22}$. La CoQ es un elemento funcional en las membranas de las células con una acción antioxidante y ayuda a regenerar el estado de reducción. La CoQ también provee un control importante en los canales de intercambio de las membranas, y su biosíntesis en los mitocondrias y el retículo endoplasmático está inhibida por las estatinas ${ }^{23}$.

\section{Estudio JUPITER}

¿El estudio "Justification for the Use of Statins in Prevention: an Intervention Trial Evaluating Rosuvastatin (JUPITER)"24 es astronómica como ciencia o a nivel de mercadeo? Este concluyó que los pacientes que tenían LDL menores a $130 \mathrm{mg} /$ dl y PCRas de 2,0 mg/l o más, tenían menores episodios de eventos coronarios (EC) con rosuvastatina a una dosis de $20 \mathrm{mg} / \mathrm{dl}$. Fue un estudio grande, que confirmó el papel de la PCRas en EC y, a su vez, apoyó el dicho: "mientras más bajo, mejor", en relación con las $\mathrm{LDL}^{25}$. No obstante, hace falta un nuevo trabajo científico y de gran envergadura. Todas las estatinas disminuyen las $\mathrm{LDL}^{14}$ y la PCRas ${ }^{26}$ en proporción a su potencia. No es necesario administrar las estatinas a altas dosis para disminuir la PCRas; se puede mantener la dosis baja para reducir el riesgo de una miopatía ${ }^{27}$ y todavía alcanzar una disminución significativa de la PCRas con la adición de ezetimiba ${ }^{26}$ y así lograr que los valores de las LDL sean menores de $70 \mathrm{mg} / \mathrm{dl}$ en los pacientes con alto riesgo para EC. Posiblemente, se logre al cambiar a rosuvastatina a la dosis diaria de $20 \mathrm{mg}$, o aun $40 \mathrm{mg}$, la apropiada para un paciente con una hipercolesterolemia alta y difícil de controlar, pero tendrá un mayor riesgo de presentar una miopatía comparado con una dosis más baja de esta estatina o de las otras ${ }^{27}$. A ningún paciente se le debe cambiar o escoger la estatina por razones comerciales o debidas a los seguros médicos. Esperemos que un buen estudio, que añada pruebas para la prevención de EC, siga esta línea.

\section{Conclusiones}

El tipo de fármacos conocido como estatinas es imprescindible para la práctica medica moderna, ya sea para la prevención o tratamiento de las enfermedades CVs. Las mayores contribuciones de este tipo de medicamentos, con una marcada disminución de las LDL y de sus beneficiosos efectos pleotrópicos, están bien establecidas -incluyendo el número de muertes, infartos del miocardio y de revascularización coronaria. Sin embargo, como todo tratamiento, existen limitaciones y efectos secundarios que es importante saber manejar y tratar con cuidado. Así la balanza se inclina a favor de su uso en los pacientes con alto riesgo CV. El uso apropiado y experto de las estatinas ha marcado una gran diferencia en la vida de muchos pacientes CV. A su vez, se hacen imprescindibles nuevos conocimientos, nuevos fármacos y experiencia para alterar las complejidades no solucionadas en lo referente a la prevención de las enfermedades CVs. 


\section{Referencias}

1. Staffa JA, Chang J, Green L. Cerivastatin and reports of fatal rhabdomyolysis. N Engl J Med 2002; 346: 539-40.

2. FDA Approved Drug Products: Zocor. http://www. accessdata.fda.gov/scripts/cder/drugsatfda/index.cfm? fuseaction=Search.DrugDetails.

3. Altieri DC. Statins' benefits begin to sprout. J Clin Invest. 2001; 108: 365-6.

4. Shepherd J, Cobbe SM, Ford I, Isles CG, Lorimer AR, MacFarlane PW, et al. Prevention of coronary heart disease with pravastatin in men with hypercholesterolemia. West of Scotland Coronary Prevention Study Group. N Engl J Med 1995; 333: 1301-7.

5. Walmart Prescriptions Program. http://www.walmart. com/pharmacy.

6. Hyre A, Krousel-Wood MA, Muntner P, Kawasake L, DeSalvo KB. Prevalence and predictors of poor antihypertensive medication adherence in an urban health clinic setting. J Clin Hypertens 2007; 9: 179-86.

7. Fuster V. A new perspective on nonprescription statins: an opportunity for patient education and involvement. Am J Cardiol 2007; 100: 907-10.

8. Whayne TF, Jr. Nonprescription statins: not ready for prime time. Am J Cardiol 2008; 101: 745-6; author reply 46.

9. Thompson P, Clarkson P, Karas RH. Statin-associated myopathy. JAMA 2003; 289: 1681-90.

10. Nichols GA, Koro CE. Does statin therapy initiation increase the risk for myopathy? An observational study of 32,225 diabetic and nondiabetic patients. Clin Ther 2007; 29: 1761-70.

11. Bruckert E, Hayem G, Dejager S, Yau C, Begaud B. Mild to moderate muscular symptoms with high-dosage statin therapy in hyperlipidemic patients--the PRIMO study. Cardiovasc Drugs Ther 2005; 19: 403-14.

12. Harper CR, Jacobson TA. The broad spectrum of statin myopathy: from myalgia to rhabdomyolysis. Curr Opin Lipidol 2007; 18: 401-8.

13. Olsson AG, Pears J, Mc Kellar J, Mizan J, Raza A. Effect of rosuvastatin a low-density lipoprotein cholesterol in patients with hypercholesterolemia. Am J Cardiol 2001; 88: 504-8.

14. Jones PH, Davidson MH, Stein EA, Bays HE, McKenney JM, Miller E, et al. Comparison of the efficacy and safety of rosuvastatin versus atorvastatin, simvastatin, and pravastatin across doses (STELLAR ${ }^{\star}$ Trial). Am J Cardiol
2003; 92: 152-60.

15. Gagne C, Bays HE, Weiss SR, Mata P, Quinto K, Melino $\mathrm{M}$, et al. Efficacy and safety of ezetimibe added to ongoing statin therapy for treatment of patients with primary hypercholesterolemia. Am J Cardiol 2002; 90: 1084-91.

16. Cannon CP, Braunwald E, McCabe CH, Rader DJ, Rouleau JL, Belder R, et al. Intensive versus moderate lipid lowering with statins after acute coronary syndromes. N Engl J Med 2004; 350: 1495-504.

17. LaRosa J, Grundy SM, Waters DD, Shear C, Barter P, Fruchart JC, et al, for the Treating to New Targets (TNT) Investigators. Intensive lipid lowering with atorvastatin in patients with stable coronary disease. N Engl J Med 2005; 352: 1425-35.

18. Whayne TF, Jr. Is there a problem with ezetimibe or just enhanced hype? Angiology 2009; 59: 661-3.

19. Peto R, Emberson J, Landray M, Baigent C, Collins R, Clare R, et al. Analyses of cancer data from three ezetimibe trials. N Engl J Med 2008; 359: 1357-66.

20. Pierce LR, Wysowski DK, Gross TP. Myopathy and rhabdomyolysis associated with lovastatin-gemfibrozil combination therapy. JAMA 1990; 264: 71-5.

21. Stalenhoef AF. Management of mixed hyperlipidaemia. Eur Heart J 2005; 26: 856-7.

22. Caso G, Kelly P, McNurlan MA, Lawson WE. Effect of coenzyme q10 on myopathic symptoms in patients treated with statins. Am J Cardiol 2007; 99: 1409-12.

23. Marcoff L, Thompson PD. The role of coenzyme Q10 in statin-associated myopathy: a systematic review. J Am Coll Cardiol 2007; 49: 2231-7.

24. Ridker PM, Danielson E, Fonseca FA, Genest J, Gotto AM, Jr., Kastelein JJ, et al. Rosuvastatin to prevent vascular events in men and women with elevated C-reactive protein. N Engl J Med 2008; 359: 2195-207.

25. Wiviott SD, Cannon CP, Morrow DA, Ray KK, Pfeffer MA, Braunwald E. Can low-density lipoprotein be too low? The safety and efficacy of achieving very low low-density lipoprotein with intensive statin therapy: a PROVE IT-TIMI 22 substudy. J Am Coll Cardiol 2005; 46: 1411-6.

26. Sager PT, Melani L, Lipka L, Strony J, Yang B, Suresh $\mathrm{R}$, et al. Effect of coadministration of ezetimibe and simvastatin on high-sensitivity C-reactive protein. Am J Cardiol 2003; 92: 1414-8.

27. Thompson PD, Clarkson P, Karas RH. Statin-associated myopathy. JAMA 2003; 289: 1681-90. 